Biochimica et Biophysica Acta, 436 (1976) 210-221

(C) Elsevier Scientific Publishing Company, Amsterdam - Printed in The Netherlands

BBA 77315

\title{
AN ANALYSIS OF THE ADEQUACY OF THE ASYMMETRIC CARRIER MODEL FOR SUGAR TRANSPORT
}

\author{
DAVID M. FOSTER and JOHN A. JACQUEZ \\ Department of Physiology, The University of Michigan Medical School, Ann Arbor, Mich. 48109 (U.S.A)
}

(Received July 15th, 1975)

(Revised manuscript received December 22nd, 1975)

\section{SUMMARY}

In 1972, Lieb, W. R. and Stein, W. D. (Biochim. Biophys. Acta 265, 187-207) in their review of sugar transport in human erythrocytes concluded that the conventional two-state carrier model was inconsistent with the experimental data available at that time. Since then, other papers have appeared which question the validity of the model. In this paper, we give a brief derivation of the equations describing the two-state carrier model, and analyze the predictions of the model in the classical experiments, i.e. zero-trans, infinite-cis, and equilibrium exchange. We show that the estimate of the half saturation constant of $2.8 \mathrm{mM}$ for glucose at the inner face of the human red cell membrane for the infinite-cis procedure reported by Hankin, B. L., Lieb, W. R. and Stein, W. D ((1972) Biochim. Biophys. Acta 288, 114-126) is unreliable. We note that all of the other experimental findings are consistent with the asymmetric carrier model.

\section{INTRODUCTION}

In 1972, Lieb and Stein [1] reviewed the experimental data on sugar transport in the human erythrocyte and concluded that they were not consistent with the conventional carrier models. These findings have stimulated some controversy as to the validity of the carrier model as well as the rejection criteria presented in [1], by Hankin et al. [2], and by Hoare [3]. Geck [4] discusses the incompatibilities of the symmetric model and shows that they are resolved by the asymmetric model. Geck's observations are confirmed by Regen and Tarpley [5]. These authors also address themselves to the rejection criteria pointing out the error in Hoare's equation (Eqn. 1 in ref. 3) and mentioning the inconsistency between the data collected by Hankin et al. [2] and Lacko et al. [6]. Edwards [7] states that the only published data which is inconsistent with the asymmetric model is given in ref. 2 . He speculates that the estimates presented there are probably low because the extracellular glucose concentration is not truly saturating.

The rejection criteria that have been presented to date involve relationships among the Michaelis-Menten constants predicted by the carrier model for the zerotrans, infinite-cis, and equilibrium exchange experiments. The problem in applying 
these criteria is that data on influx experiments are required. Hankin et al. [2] have devised an influx experiment under the infinite-cis condition which allows them to calculate the parameters needed to apply their rejection criteria. These are then used to reject the asymmetric carrier model. Lacko et al. [6] have estimated the parameters in zero-trans influx experiments. If their estimates are used in the criteria in [1] and [2] we see that the asymmetric model is consistent with the data. Bloch [8] has studied the asymmetric features of sugar transport, and his data also satisfies the criteria in [1] and $[2]$.

It is easy to infer from the discussion above that the theoretical treatment of the two-state carrier model has been motivated by the theory of enzyme kinetics. Several authors, most notably Regen and Morgan [9], Britton [10], Sen and Widdas [11], and Miller [12] have analyzed the model from the mathematical point of view. Unfortunately, the derivation of the basic equations is not as obvious as it is in enzyme kinetics. For this reason, we begin this paper with a succinct derivation of the basic equations describing the two-state carrier model. We see that the flux equation arises naturally as the zero-order singular perturbation solution of a system of equations whose development closely parallels the usual development of the Michaelis-Menten equation. Although the final equations for the different fluxes and the form of the Michaelis-Menten constants for the classical experiments remain the same, we believe that because of the brevity of the derivation the reader can get more insight into the theoretical nature of the model. This is especially true in the equilibrium exchange case.

In the final part of the paper, we will analyze the theoretical treatment of the infinite-cis experiments in more detail. Our interest in this is motivated by the fact that the estimates for $K_{\mathrm{m}}$ and $V$ reported in [2] in the infinite-cis influx are the only data which are inconsistent with the two-state carrier model. We will show first that the equation used in [2] to estimate these numbers is far too sensitive to be reliable. We will then show that a similar method for estimating these values based on the full form of the flux equation is also too sensitive. Our conclusion is that these values for $K_{\mathrm{m}}$ and $V_{\max }$ should be disregarded.

\section{The derivation of the flux equation}

The conventional two-state carrier model is given in Fig. 1. To facilitate the comparison between our work and that given in [1] and [2], we are using the same notation.

The equation which describes the conservation of carrier in this system is

$$
T=E_{1}+E_{2}+(E S)_{1}+(E S)_{2}
$$

where $T$ is the total amount of carrier per membrane unit. The time derivatives of $E_{i}$ and $(E S)_{i}, i=1,2$ are

$$
\begin{aligned}
& \frac{d\left(E_{1}\right)}{d t}=h E_{2}+b(E S)_{1}-g E_{1}-a S_{1} E_{1} \\
& \frac{d\left(E_{2}\right)}{d t}=g E_{1}+e(E S)_{2}-h E_{2}-f S_{2} E_{2}
\end{aligned}
$$




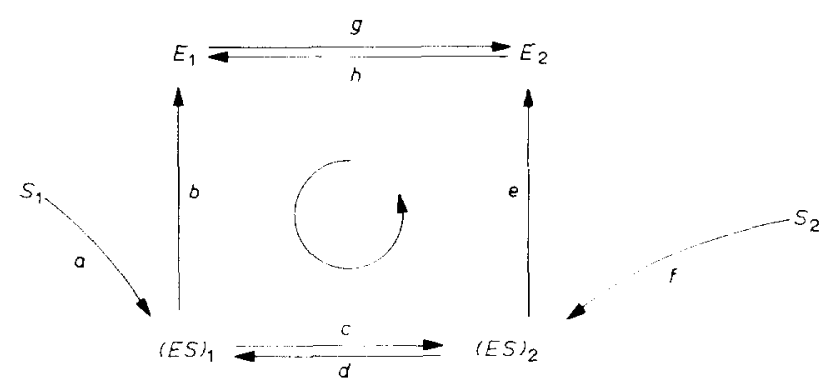

Fig. 1. Kinetic scheme for the conventional carrier model. $S_{1}$ and $S_{2}$ represent substrate concentrations in the solutions bathing sides 1 and 2 of the membrane respectively. $E_{1}$ and $E_{2}$ represent the concentrations of free carrier while $(E S)_{1}$ and $(E S)_{2}$ represent the concentrations of carrier-substrate complex at faces 1 and 2 of the membrane respectively. The rate constants are denoted by $a$ through $h$. Movement counterclockwise through the system is taken to be positive.

$$
\begin{aligned}
& \frac{d\left((E S)_{1}\right)}{d t}=a S_{1} E_{1}+d(E S)_{2}-(b+c)(E S)_{1} \\
& \frac{d\left((E S)_{2}\right)}{d t}=f S_{2} E_{2}+c(E S)_{1}-(d+e)(E S)_{2}
\end{aligned}
$$

The standard assumption used to solve these equations is that the concentrations of the different carrier species do not change much, i.e. upon the addition of substrate they rapidly assume values which then change very little over a substantial portion of the transport process. Consequently, the derivatives in Eqn. 2 are set equal to zero. This "pseudo-stationary state" assumption is also used in solving the differential equations for enzyme reactions. Mathematically, it gives the zero order singular perturbation solution of the system. With this assumption, Eqns. 1 and 2 become a system of linear equations in $E_{1}, E_{2},(E S)_{1}$, and $(E S)_{2}$. Solving, we obtain

$$
\begin{gathered}
E_{1}=-\frac{T}{D}\left\{h(b d+b e+c e)+b d f S_{2}\right\} \\
E_{2}=-\frac{T}{D}\left\{g(b d+b e+c e)+a c e S_{1}\right\} \\
(E S)_{1}=-\frac{T}{D}\left\{a h(d+e) S_{1}+d f g S_{2}+a d f S_{1} S_{2}\right\} \\
(E S)_{2}=-\frac{T}{D}\left\{f g(b+c) S_{2}+a c h S_{1}+a c f S_{1} S_{2}\right\} \\
D=-\left\{(h+g)[b(d+e)+c e]+[g(b+c+d)+b d] f S_{2}\right. \\
\left.\quad+[h(c+d+e)+c e) a S_{1}+(c+d) a f S_{1} S_{2}\right\} .
\end{gathered}
$$

In the model, the possible one way fluxes through each step are

$$
\begin{array}{ll}
\vec{J}_{1}=a E_{1} S_{1} & \overleftarrow{J}_{1}=-b(E S)_{1} \\
\vec{J}_{2}=c(E S)_{1} & \overleftarrow{J}_{2}=-d(E S)_{2}
\end{array}
$$


$\vec{J}_{3}=e(E S)_{2} \quad \vec{J}_{3}=-f S_{2} E_{2}$

$\vec{J}_{4}=h E_{2} \quad \overleftarrow{J}_{4}=-g E_{1}$.

Here, $\rightarrow$ represents positive flux which is taken as the direction going counterclockwise around the reaction path in Fig. 1 while $\leftarrow$ represents movement in the opposite direction. Therefore, in the pseudo-stationary state we have

$\vec{J}_{\mathbf{k}}+\overleftarrow{J}_{\mathbf{k}}=-\frac{T}{D}\left(a^{a c e h} S_{1}-b d f g S_{2}\right), \quad k=1,2,3,4$.

If we use the fact that for uncharged substrate $a c e h=b d f g$ (see Britton [10]), and if we write $J_{\mathrm{n}}$ for the net flux through the system in the pseudostationary state, we have

$J_{\mathrm{n}}=\vec{J}_{\mathrm{k}}+\breve{J}_{\mathrm{k}}=-\frac{\operatorname{aceh} T}{D}\left(S_{1}-S_{2}\right), \quad k=1,2,3,4$.

This is the same equation as Eqn. 1 in ref. 2, the derivation of which is attributed to Britton [10]. It is also the same as Eqn. 6 in ref. 9. One should notice that this equation has been derived from the assumption that the net flux of carrier through each step is equal to the overall transport rate. This assumption depends on the validity of the pseudo-stationary state assumption.

\section{Analysis of the two-state carrier model}

We will now consider the zero-trans, infinite-cis, and equilibrium exchange experiments where we assume movement of substrate from side 2 to side 1 . In presenting the results, we will list those for movement from side 1 to side 2 as well with the understanding that they are found in an analogous manner to those actually derived.

When measuring the appearance or disappearance of substrate in this situation, we are examining the reaction given by Eqn. 6:

$S_{1}+E_{1} \rightleftarrows(E S)_{1}$.

Of the steps shown in Fig. 1, this is the step that involves movement of substrate from the environment to the cell or in the reverse direction and hence is the step observed experimentally. Therefore, the correct form of the net flux equation is

$J_{\mathrm{n}}=a E_{1} S_{1}-b(E S)_{1}$.

Zero-trans. In the zero-trans experiments, $S_{1}$ is kept equal to zero while $S_{2}$ is varied. Thus

$J_{2 \rightarrow 1}^{\mathrm{zt}}=\bar{J}=-b(E S)_{1}=\frac{b T}{D}\left\{a h(d+e) S_{1}+d f g S_{2}+a d f S_{1} S_{2}\right\}$.

Setting $S_{1}=0$ we can write this in Michaelis-Menten form as

$J_{2 \rightarrow 1}^{\mathrm{zt}}=-\frac{V_{2 \rightarrow 1}^{\mathrm{zt}} S_{2}}{K_{2 \rightarrow 1}^{\mathrm{zt}}+S_{2}}$.

From Eqn. 9 and the corresponding equation for $J_{1 \rightarrow 2}{ }^{\text {zt }}$ we obtain 


$$
\begin{aligned}
K_{2 \rightarrow 1}^{\mathrm{zt}}=\frac{(g+h)[e(b+c)+b d]}{[g(b+c)+d(b+g)] f} ; & K_{1 \rightarrow 2}^{\mathrm{zt}}=\frac{(g+h)[e(b+c)+b d]}{[h(d+e)+c(e+h)] a} \\
V_{2 \rightarrow 1}^{\mathrm{zt}} & =\frac{b d g T}{g(b+c)+d(b+g)} ; \quad V_{1 \rightarrow 2}^{\mathrm{zt}}=\frac{c e h T}{h(d+e)+c(e+h)} .
\end{aligned}
$$

The estimates for $V_{2 \rightarrow 1}{ }^{2 t}$ and $K_{2 \rightarrow 1}{ }^{2 t}$ are obtained from an inverse plot. The accuracy of these estimates depends upon $S_{1}$ being equal to zero. Thus in the experimental design provisions for maintaining $S_{1}$ at or near zero must be taken into account.

Infinite-cis. In the infinite-cis experiments, the assumption is made that $S_{2}$ far exceeds the saturating value at face 2 , and $S_{1}$ is varied. In this case, Eqn. 7 cannot be written in Michaelis-Menten form. We can, however, derive analogs of the MichaelisMenten constants. The assumption is made that the maximal flux will occur when $S_{1}$ is zero, thus $V_{2 \rightarrow 1}$ ic $=V_{2 \rightarrow 1}{ }^{\text {zt }}$. To find $K_{2 \rightarrow 1}$ ic, we simply solve $J_{2 \rightarrow 1}=\frac{1}{2} V_{2 \rightarrow 1}$ ic for $S_{1}$. If we do this, we find that $S_{1}$ is a quotient of two polynomials, both quadratic in $S_{2}$. To find $S_{1}$ as an expression involving the rate constants, we let $S_{2} \rightarrow \infty$. Collecting our results, we have

$$
\begin{aligned}
& V_{2 \rightarrow 1}^{\mathrm{ic}}=V_{2 \rightarrow 1}^{\mathrm{zt}} \quad V_{1 \rightarrow 2}^{\mathrm{ic}}=V_{1 \rightarrow 2}^{\mathrm{zt}} \\
& K_{2 \rightarrow 1}^{\mathrm{ic}}=\frac{g(b+c)+d(b+g)}{a(c+d)} \quad K_{1 \rightarrow 2}^{\mathrm{ic}}=\frac{h(d+e)+c(e+h)}{f(c+d)} .
\end{aligned}
$$

The problem here involves letting $S_{2} \rightarrow \infty$. Experimentally, we must have $S_{2}$ far enough above the saturating value at face 2 so that the expressions for the $K^{\text {ic }}$ are close approximations to the solutions of $J=\frac{1}{2} V^{\mathrm{ic}}$.

Equilibrium exchange. In this case, we have a true steady state where $S_{1}=S_{2}$ $=\bar{S}$, and $J_{\mathrm{n}}=0$. Thus suppose that at time zero the cells are in an overall steady state, and we have specific activities $\alpha_{2}$ of substrate at face $2, \alpha_{1}$ at face 1 . If $\beta_{1}$ and $\beta_{2}$ are the specific activities of $(E S)_{1}$ and $(E S)_{2}$ respectively, we have

$$
\begin{aligned}
& \frac{d\left(\beta_{2}(E S)_{2}\right)}{d t}=\alpha_{2} f E_{2} \tilde{S}+c \beta_{1}(E S)_{1}-(d+e) \beta_{2}(E S)_{2} \\
& \frac{d\left(\beta_{1}(E S)_{1}\right)}{d t}=\alpha_{1} a E_{1} \bar{S}-(b+c) \beta_{1}(E S)_{1}+d \beta_{2}(E S)_{2} .
\end{aligned}
$$

If the pseudo-stationary state assumption is valid for the kinetics of this system, it applies to the tracer as well, hence we set the derivatives in Eqn. 12 equal to zero. If, in addition, we assume $\alpha_{2}$ is constant and $\alpha_{1}$ is zero throughout the experiment, we can solve the resulting system for $\beta_{1}$ and $\beta_{2}$ obtaining

$$
\begin{aligned}
& \beta_{1}=\frac{\alpha_{2} d f E_{2} \bar{S}}{(b d+b e+c e)(E S)_{1}} \\
& \beta_{2}=\frac{\alpha_{2}(b+c) f E_{2} \bar{S}}{(b e+b d+c e)(E S)_{2}} .
\end{aligned}
$$

Now the tracer movement which is measured is proportional to $\vec{J}_{1}$ or $\bar{J}_{1}$ depending 
upon which side has the tracer initially. In the case when Eqn. 13 holds, the flux of measured label is

$\overleftarrow{J}_{1}^{*}=\beta_{1} \overleftarrow{J}_{1}=-\frac{\alpha_{2} b d f E_{2} \bar{S}}{b d+b e+c e}$.

Writing this in Michaelis-Menten form, we have

$\bar{J}^{*}=\frac{V_{2 \rightarrow 1}^{*} \bar{S}}{K_{2 \rightarrow 1}^{\mathrm{ee}}+\tilde{S}}$.

Eqn. 15 is easily derived using a limit argument, but it can also be derived by algebraically manipulating Eqn. 14. Since $V_{2 \rightarrow 1}{ }^{\mathrm{cc}}=\left(1 / \alpha_{2}\right) V_{2 \rightarrow 1}{ }^{*}$, we have

$$
\begin{aligned}
& V^{\mathrm{ee}}=V_{2 \rightarrow 1}^{\mathrm{ee}}=V_{1 \rightarrow 2}^{\mathrm{ee}}=\frac{T b c d e}{(c+d)[e(b+c)+b d]} \\
& K^{\mathrm{ee}}=K_{2 \rightarrow 1}^{\mathrm{ee}}=K_{1 \rightarrow 2}^{\mathrm{ee}}=\frac{b d(g+h)}{a h(c+d)} .
\end{aligned}
$$

In doing the equilibrium exchange experiments, the cells are first allowed to equilibrate with a given concentration of glucose. A small amount of tracer is then added, and it is the movement of tracer that is measured. The theoretical considerations to be kept in mind when designing the experiment involve the system being in a true steady state and the restrictions on the specific activities $\alpha_{i}, i=1,2$, of substrate.

The development which we have just given is based upon the fact that what is being measured is the movement of a small amount of tracer in a system that is in an overall steady state, i.e. $\bar{S}=S_{1}=S_{2}$. It is interesting to note that if instead of doing this analysis we set $\bar{S}=S_{1}=S_{2}$ in $\bar{J}_{1}$ we obtain a flux which is different from that given by Eqn. 16. Indeed in this case

$$
\bar{J}_{1}=\frac{\frac{b d T}{c+d} \bar{S}}{\frac{b d(g+h)}{a h(c+d)}+\bar{S}}
$$

The $K_{\mathrm{m}}$ is the same and the $V_{\max }$ differs from that in Eqn. 16 by the factor $1 /(1+(b / c)+(b d / c e))$.

\section{The infinite-cis influx experiments}

As we mentioned in the introduction, in order to apply the rejection criteria given in refs. 1 and 2, we must have information on influx experiments in either the infinite-cis or zero-trans case in order to calculate

$Q=\frac{K_{2 \rightarrow 1}^{\mathrm{ic}}}{K_{1 \rightarrow 2}^{\mathrm{ic}}}=\frac{K_{1 \rightarrow 2}^{\mathrm{zt}}}{K_{2 \rightarrow 1}^{\mathrm{zt}}}=\frac{V_{1 \rightarrow 2}^{\mathrm{zt}}}{V_{2 \rightarrow 1}^{\mathrm{zt}}}$.

$Q$ is a measure of the asymmetry of the system, i.e. if the system is not symmetric, we would expect $Q$ to be different from unity. Bloch [8] has reported values of $Q$ for 
glucose equal to 20 at $7{ }^{\circ} \mathrm{C}$ and 10 at $37^{\circ} \mathrm{C}$. If we combine the zero-trans influx data of Lacko et al. [6] with the zero-trans efflux data of Karlish et al. [13], we find a value of $Q$ of approximately 15.5 for glucose at $20^{\circ} \mathrm{C}$. In ref. 2 , the following equation is derived:

$Q=\frac{K^{\mathrm{ec}}}{K_{1 \rightarrow 2}^{\mathrm{ic}}}+\frac{K_{1 \rightarrow 2}^{\mathrm{zt}}}{K^{\mathrm{ee}}}-1$.

Notice that this expression for $Q$ involves data from efflux experiments but not influx. In [2] a value of $Q$ equal to 17.6 for glucose at $20^{\circ} \mathrm{C}$ is reported using Eqn. 19. Eqn. 19 can also be used to find a lower bound for $Q$. Indeed Eqn. 19 can be rewritten as a quadratic in $K^{\text {ee }}$ and the resulting discriminant analyzed. Doing so, we find

$Q \geqq 2\left[\frac{K_{1 \rightarrow 2}^{\mathrm{zt}}}{K_{1 \rightarrow 2}^{\mathrm{ic}}}\right]^{\frac{1}{2}}-1$.

In ref. 2 the value of the lower bound reported is $6.5 \pm 0.5$, i.e. $Q \geqslant 6.5$.

In their review of 1972, Lieb and Stein [1] analyzed the predictions of the symmetric carrier model and, on the basis of efflux experiments alone, showed that these were inconsistent with the experimental data which was available in the literature. The rejection of the symmetric model means that $Q$ must be different from one. Therefore, we have to question the statement given later in ref. 1 to the effect that $Q$ is not significantly different from unity and certainly not greater than 3 for glucose at $20^{\circ} \mathrm{C}$.

In ref. 2, a value of $Q$ equal to $1.6 \pm 0.3$ was reported having been calculated as the ratios $K_{2 \rightarrow 1}$ ic $/ K_{1 \rightarrow 2}$ ic. Given the fact that there is close agreement among the values for $K_{1 \rightarrow 2}$ ic reported in the literature (see refs. 2 or 1) and all other reported values of $Q$ reported lie in the range from 10 to 20 , we are led to suspect the calculations of $K_{2 \rightarrow 1}$ ic given in ref. 2 . Indeed, either this estimate is correct, a result which would cause one to question the validity of the two-state carrier model, or it is incorrect, a result which would remove from the literature the only set of data which are inconsistent with the model. In this section, we will study the theoretical nature of the problem.

To facilitate the comparison between what follows and that given in ref. 2 , we list and adopt their notation.

$N=$ cellular glucose concentration at time $t$ in $\mathrm{mmol} / \mathrm{cell}$ unit.

$P=$ osmolarity of non-penetrating salts in both the extracellular solution and in the isotonic cells.

$V_{\text {rel }}=$ liters of cell water per cell unit at time $t$.

$v=V_{2 \rightarrow 1}$ ic.

$K=K_{2 \rightarrow 1}$ ic.

$k=K_{2 \rightarrow 1}{ }^{\mathrm{zt}}$.

Here a cell unit is that number of cells whose solvent water volume is one liter under isotonic conditions. Using this notation, we may write Eqn. 7 as

$J_{2 \rightarrow 1}=\frac{v\left\{1-S_{1} / S_{2}\right\}}{1+\frac{k}{S_{2}}+S_{1}\left\{\frac{1}{K}+\frac{1}{Q S_{2}}\right\}}$. 
In the infinite-cis case, i.e. letting $S_{2} \rightarrow \infty$, and writing $J_{2 \rightarrow 1}{ }^{\infty}$ to denote $J_{2 \rightarrow 1}$ in this case, we obtain

$J_{2 \rightarrow 1}^{*}=\frac{v K}{K+S_{1}}$.

This shows, incidently, that Eqn. 7 cannot be written in Michaelis-Menten form in this situation. The estimates of $v$ and $K$, however, can be obtained by plotting $1 / J_{2 \rightarrow 1}{ }^{\infty}$ as a linear function of $S_{1}$. Notice that Eqns. 20 and 21 are the same, mutatis mutandis, as Eqns. 8 and 23 in ref. 2.

The starting point for the theoretical development in ref. 2 is Eqn. 22 which we can rewrite

$$
\frac{d N}{d t}=\frac{v K}{K+N / V_{\mathrm{rel}}}
$$

Notice that $S_{2}$ does not appear in this expression having been eliminated in passing from Eqn. 21 to 22.

Hankin et al. next assume that the cells are always in osmotic equilibrium with the extracellular solution and obtain

$P+S_{2}=\begin{gathered}P+N \\ V_{\mathrm{rcl}}\end{gathered}$

This equation is solved for $1 / V_{\text {rel }}$ and the result substituted into Eqn. 22 to obtain

$$
\frac{d N}{d t}=\frac{v K}{K+\frac{N\left(P+S_{2}\right)}{P+N}}
$$

This reintroduces $S_{2}$ into the equation thereby neglecting any interplay that might occur between the terms containing $S_{2}$ that were eliminated and that which has just been reintroduced. Furthermore, Eqn. 24 is wrong dimensionally, and this carries over into the remainder of the derivation. To put Eqn. 24 into the proper dimensional form, let $V_{0}$ equal $V_{\text {re } 1}$ under isotonic conditions. Then $V_{0}=1$ liter cell water/cell unit. The correct form for Eqn. 24 becomes 24':

$P+S_{2}=\frac{P V_{0}+N}{V_{\mathrm{rcl}}}$

Since $V_{0}=1$, Eqn. 24 is correct numerically hence we do not carry $V_{0}$ explicitly in what follows.

How were the estimates of $K$ computed? In ref. 2, Eqn. 25 was integrated under the assumptions that $S_{2}$ was constant, $N=0$ when $t=0$, and $N=N$ when $t=t$ giving

$K N+\left(P+S_{2}\right)\left[N-P \ln \left(1+\frac{N}{P}\right)\right]=v K t$.

'This was rewritten 
TABLE I

EFFECT OF ROUND-OFF IN SLOPE, $\sigma$, AND OF AN INCREASE IN SLOPE ON ESTIMATED $K$

\begin{tabular}{|c|c|c|c|c|c|c|c|}
\hline \multicolumn{2}{|c|}{ Data in ref. 2} & \multicolumn{2}{|c|}{ Round-off } & \multicolumn{2}{|c|}{$2 \%$ increase in $\sigma$} & \multicolumn{2}{|c|}{$5 \%$ increase in $\sigma$} \\
\hline $\begin{array}{l}K \\
(\mathrm{mM})\end{array}$ & $\begin{array}{l}\sigma \cdot 10^{3} \\
\left((\mathrm{mM})^{-1}\right)\end{array}$ & $\sigma \cdot 10^{3}$ & $K$ & $\sigma \cdot 10^{3}$ & $K$ & $\sigma \cdot 10^{3}$ & $K$ \\
\hline 2.2 & 3.245 & 3.25 & 2.8 & 3.310 & 9.64 & 3.407 & 20.81 \\
\hline 0.5 & 3.230 & 3.23 & 0.5 & 3.295 & 7.91 & 3.392 & 19.03 \\
\hline 2.8 & 3.250 & 3.25 & 2.8 & 3.315 & 10.26 & 3.413 & 21.44 \\
\hline 3.7 & 3.258 & 3.26 & 3.9 & 3.323 & 11.17 & 3.421 & 22.39 \\
\hline 3.6 & 3.257 & 3.26 & 3.9 & 3.322 & 11.07 & 3.420 & 22.28 \\
\hline 1.3 & 3.237 & 3.24 & 1.6 & 3.302 & 8.73 & 3.399 & 19.87 \\
\hline Av. 2.4 & 3.247 & 3.25 & 2.6 & 3.311 & 9.8 & 3.409 & 20.97 \\
\hline
\end{tabular}

$$
\frac{1}{t} \ln \left(1+\frac{N}{P}\right)=\frac{K+P+S_{2}}{P\left(P+S_{2}\right)} \frac{N}{t}-\frac{v K}{P\left(P+S_{2}\right)} .
$$

To compute $K,(1 / t) \ln (1+N / P)$ was plotted against $N / t$ and the slope was set equal to $\left(K+P+S_{2}\right) /\left(P\left(P+S_{2}\right)\right)$. Once $K$ was known, $v$ was found from the intercept of this line. Because this involves an extrapolation outside the range of the experimental points, we will not investigate the problems with $v$.

Let us denote by $\sigma$ the slope of this line. Then

$K=\left(P+S_{2}\right)(P \sigma-1)$.

In most of the experiments reported in ref. $2, P=310 \mathrm{mM}$ and $S_{2}=60 \mathrm{mM}$ whence

$K=114700 \sigma-370$.

Two facts are readily apparent. One is that for $K$ to be small, $\sigma$ must just exceed $1 / 310=3.226 \cdot 10^{-3}$, and the other is that $K$ will be extremely sensitive to even small errors in estimates for $\sigma$.

We are now in a position to show why the data presented in ref. 2 in conjunction with Eqn. 29 produce unreliable estimates for $K$. Let us begin with the data given in Table II in ref. 2. For the first six experiments, $S_{2}=60 \mathrm{mM}$ and $P=310 \mathrm{mM}$, and the average $K$ for these is 2.4. For each of the individual $K$ values the corresponding $\sigma$ can be calculated from Eqn. 29. These are summarized in the first two columns of Table $I$. Notice that we must record $\sigma-4$ significant figures to produce the correct value of $K$.

Our first study involves the question of what happens to $K$ as a result of small changes in $\sigma$. The third and fourth columns summarize what happens as a result of round-off error. Notice that although the average $K$ rises, it is still well below the value predicted by the model. The last four columns summarize what happens as a result of a $2 \%$ and $5 \%$ increase in each $\sigma$. A $2 \%$ increase produces an average $K$ of $9.8 ;$ a $5 \%$ increase produces an average $K$ of 21 . If we return to Eqn. 20, we see that $Q \geqslant 6.5$ together with $K_{2 \rightarrow 1}$ ic $=1.8$ implies $K \geqslant 11.7$. Thus a $2 \%$ increase in each $\sigma$ brings the average $K$ to a value just below the cutoff point whereas a $5 \%$ increase produces an average $K$ well above this limit. 
TABLE II

DATA USED TO ESTIMATE SLOPE, $\sigma$. (FROM REF. 2)

\begin{tabular}{lccc}
$t(\min )$ & $N(t-S . D.) \mathrm{mmol} /$ cell unit & $N / t$ & $1 / t \ln (1+N / 310) \cdot 10^{2}$ \\
\hline 0.17 & 3.8 .10 .3 & $22(22.4)$ & $7.2(7.17)$ \\
0.33 & $5.8+0.5$ & $18(17.6)$ & $5.6(5.62)$ \\
0.50 & $7.4-0.6$ & $15(14.8)$ & $4.7(4.72)$ \\
0.67 & $8.6-0.7$ & $13(12.8)$ & $4.1(4.08)$ \\
\hline
\end{tabular}

The next step in our study involves the question of whether or not such changes are possible in $\sigma$ as a result of experimental error. To investigate this question we turn to the data presented in Fig. 2 of ref. 2. The values for $t$ and $N$ which we have taken from the figure are listed in the first two columns of Table II. Notice that we have retained two significant figures.

Next we investigated how sensitive $N / t$ and $(1 / t)[\ln (1+N / P)]$ are to small changes in $N, t$ and $P$. Thus we calculated these values for $P=305 \mathrm{mM}, 310 \mathrm{mM}$, $315 \mathrm{mM}, t=0.16 \mathrm{~min}, 0.17 \mathrm{~min}$, and $N=3.75 \mathrm{mM} /$ cell unit, $3.8 \mathrm{mM} /$ cell unit, $3.83 \mathrm{mM} /$ cell unit, and $3.85 \mathrm{mM} /$ cell unit. We found a sizable variation in both $N / t$ and $(1 / t)[\ln (1+N / P)]$ with the differences occurring in the second significant figure. Because of this, we decided to use $P=310 \mathrm{mM}$ and to calculate $N / t$ and $(1 / t)[\mathrm{ln}$ $(1-N / P)]$ from the data listed in the first two columns of Table II retaining two figures. These values are listed in the final two columns with the respective numbers to three significant figures given in parentheses.

We then did a least squares fit to the data in Table II that was correct to two significant figures and obtained the line,

$\frac{1}{t} \ln \left(1+\frac{N}{310}\right)=3.435 \cdot 10^{-3} \frac{N}{t}-4.391 \cdot 10^{-3}$.

The value for $\sigma=3.435 \cdot 10^{-3}$ when substituted into Eq11. 29 gives an estimated $K$ equal to 24. Since this value is considerably different from that given in [2], we decided also to fit the data that were correct to three significant figures (in parentheses in Table II). Then, the least squares fit was,

${ }_{t}^{1} \ln \left(1+\frac{N}{310}\right)=3.220 \cdot 10^{-3} \frac{N}{t}-4.361 \cdot 10^{-4}$.

Here, $\sigma=3.220 \cdot 10^{-3}$ and when this is substituted into Eqn. $29, K=-6.7$ !

Finally, we did some least squares fits where the points chosen reflected $1 \%$ to $2 \%$ errors in $N, t$ and $P$ and found values of $K$ ranging numerically between -15 and 30. Thus it is clear that the changes in $\sigma$ listed in Table I are indeed possible, and may be smaller than what may actually occur. Therefore, the estimates given in ref. 2 are unreliable and no conclusion based upon them concerning the two-state carrier model is possible.

The final question to which we addressed ourselves concerns the possibility of salvaging the data generated experimentally in [2]. Our concern here is that the starting point for the theoretical development in [2], namely Eqn. 22, is a poor choice. 
A more fitting starting point is Eqn. 21, for using Eqn. 21 we do not have the problem with $S_{2}$ mentioned above.

If we substitute for $1 / V_{\text {rel }}$, we can rewrite Eqn. 21 as

$$
\frac{d N}{d t}=\frac{v\left\{\frac{P\left(S_{2}-N\right)}{(P+N)\left(S_{2}+k\right)}\right\} K\left\{\begin{array}{c}
S_{2}+k \\
S_{2}
\end{array}\right\}\left\{\frac{Q S_{2}}{Q S_{2}+K}\right\}}{K\left\{\frac{S_{2}+k}{S_{2}}\right\}\left\{\frac{Q S_{2}}{Q S_{2}+K}\right\}+\frac{N\left(P+S_{2}\right)}{P+N}} .
$$

If we set

$\alpha=\frac{P\left(S_{2}-N\right)}{(P+N)\left(S_{2}+k\right)}$

and

$\beta=\frac{S_{2}+k}{S_{2}} \cdot \frac{Q S_{2}}{Q S_{2}+K}$

then the question as to whether or not Eqn. 30 can be approximated by Eqn. 25 will be answered once we know how close $\alpha$ and $\beta$ are to unity.

If $Q$ lies somewhere around 10 and $S_{2}$ is $60 \mathrm{mM}$, then it is clear that $\beta \approx 1$. Thus the estimates of $K$ from either equation should be in reasonable agreement. However, $\alpha$ depends both on $S_{2}$ and $N$. When $N=0, \alpha \approx 1$ but as $N$ increases, $\alpha$ decreases to values on the order of 0.8 . Any error will of course be compounded by integration, so we would expect a deviation in the values of $v$ estimated from the two equations.

The point, however, is not so much this analysis but the fact that we can integrate Eqn. 30 using the same assumptions under which Eqn. 25 was integrated. Doing so, we obtain

$$
\begin{gathered}
v K t=-\frac{K Q\left(S_{2}+k\right)\left(P+S_{2}\right)+S_{2}\left(P+S_{2}\right)\left(Q S_{2}+K\right)}{P Q} \ln \left(1-\frac{N}{S_{2}}\right) \\
-\frac{\left(P+S_{2}\right)\left(Q S_{2}+K\right)+K Q\left(S_{2}+k\right)}{P Q} N .
\end{gathered}
$$

Motivated by ref. 2 we rewrite this

$$
\begin{aligned}
\frac{1}{t} \ln \left(1-\frac{N}{S_{2}}\right)= & -\frac{\left(P+S_{2}\right)\left(Q S_{2}+K\right)+K Q\left(S_{2}+k\right)}{K Q\left(S_{2}+k\right)\left(P+S_{2}\right)+S_{2}\left(P+S_{2}\right)\left(Q S_{2}+K\right)} \frac{N}{t} \\
& -\frac{P Q v K}{K Q\left(S_{2}+k\right)\left(P+S_{2}\right)+S_{2}\left(P+S_{2}\right)\left(Q S_{2}+K\right)} .
\end{aligned}
$$

We can then plot $1 / t \ln \left(1-N / S_{2}\right)$ vs $N / t$. If $\sigma^{*}$ is the slope of this line, then

$$
\sigma^{*}=-\frac{\left(P+S_{2}\right)\left(Q S_{2}+K\right)+K Q\left(S_{2}+k\right)}{K Q\left(S_{2}+k\right)\left(P+S_{2}\right)+S_{2}\left(P+S_{2}\right)\left(Q S_{2}+K\right)} .
$$


If we set $Q=K / K_{1 \rightarrow 2}{ }^{\text {ic }}, P=310 \mathrm{mM}, S_{2}=60 \mathrm{mM}, K_{1 \rightarrow 2}$ ic $=1.8 \mathrm{mM}$ and $k=1.6$ $\mathrm{mM}$, we obtain

$K\left\{\left(12662 \sigma^{*}+34.22\right) K+762000 \sigma^{*}+12703\right\}=0$

which gives

$$
K=-\frac{762000 \sigma^{*}+12703}{12662 \sigma^{*}+34.22} \text {. }
$$

For some of the data which we used previously to estimate $K$ we replotted according to this scheme and found that the new estimate of $K$ was usually larger but reasonably close to the estimate according to the scheme in ref. 2 . This confirms our statement that $\beta \approx 1$ should produce estimates of $K$ that are close for either scheme. The important point, however, is that this equation is just as sensitive as Eqn. 29 to small changes in $\sigma^{*}$, i.e. it too cannot be used as a reliable way to estimate $K$.

As a result of the analysis presented in this section, we are forced to reject the estimates of $K$ given in ref. 2.

\section{ACKNOWLEDGEMENT}

This work was supported by a postdoctoral fellowship to the senior author, GM 00110 , from the National Institutes of Health.

\section{REFERENCES}

1 Lieb, W. R. and Stein, W. D. (1972) Biochim. Biophys. Acta 265, 187-207

2 Hankin, B. L., Lieb, W. R. and Stein, W. D. (1972) Biochim. Biophys. Acta 288, 114-126

3 Hoare, D. G. (1972) Biochem. J. 127, 62

4 Geck, P. (1971) Biochim. Biophys. Acta 241, 462-472

5 Regen, D. M. and Tarpley, H. L. (1974) Biochim. Biophys. Acta 339, 218-233

6 Lacko, L., Wittke, B. and Kromphardt, H. (1972) Eur. J. Biochem. 25, 447-454

7 Edwards, P. A. W. (1974) Biochim. Biophys. Acta 345, 373-386

8 Bloch, R. (1975) J. Biol. Chem. 249, 3543-3550

9 Regen, D. M. and Morgan, H. E. (1964) Biochim. Biophys. Acta 79, 151-166

10 Britton, H. G. (1965) J. Theoret. Biol. 10, 28-52

11 Sen, A. K. and Widdas, W. F. (1962) J. Physiol. Lond. 160, 392-403

12 Miller, D. M. (1968) Biophys. J. 8, 1339-1352

13 Karlish, S. J. D., Ram, W. R. and Stein, W. D. (1972) Biochim. Biophys. Acta 255, 126 